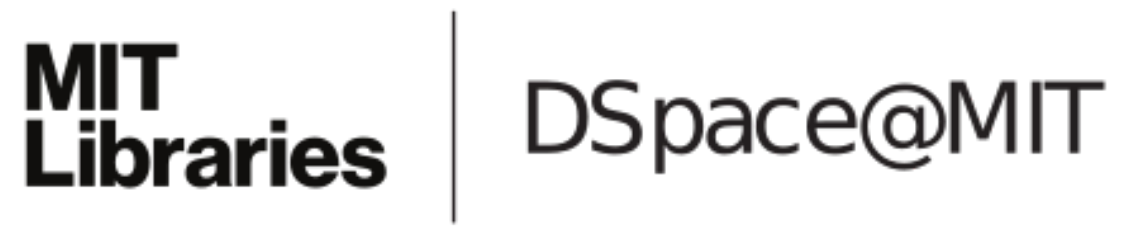

\author{
MIT Open Access Articles
}

Low-cost diaper wetness detection using hydrogel-based RFID tags

The MIT Faculty has made this article openly available. Please share how this access benefits you. Your story matters.

Citation: Sen, Pankhur et al. “Low-cost diaper wetness detection using hydrogel-based RFID tags.” IEEE Sensors Journal, 20, 6 (March 2020): 3293 - 3302 (c) 2020 The Author(s)

As Published: 10.1109/JSEN.2019.2954746

Publisher: Institute of Electrical and Electronics Engineers (IEEE)

Persistent URL: https://hdl.handle.net/1721.1/127791

Version: Author's final manuscript: final author's manuscript post peer review, without publisher's formatting or copy editing

Terms of use: Creative Commons Attribution-Noncommercial-Share Alike 


\title{
Low-cost diaper wetness detection using hydrogel-based RFID tags
}

\author{
Pankhuri Sen, Sai Nithin R. Kantareddy, Rahul Bhattacharyya, Sanjay E. Sarma ${ }^{1}$ and Joshua E. Siegel ${ }^{2}$ \\ ${ }^{1}$ Massachusetts Institute of Technology Auto-ID Labs, Department of Mechanical Engineering \\ Email: \{senp, nithin, rahul_b, sesarma\}@mit.edu \\ ${ }^{2}$ Michigan State University, Department of Computer Science and Engineering \\ Email: jsiegel@msu.edu
}

\begin{abstract}
There is an opportunity to utilize wearable, consumer-oriented sensors for self-health monitoring and preventative medicine. Disposable diaper with built-in low-cost, disposable moisture sensors is one such product. Diaper users include infants, elderly, disabled individuals, and hospital patients. Event-based alerting can enhance care of this population by improving incontinence management, preventing rashes and infections, and avoiding embarrassment. Collected data can be used to optimize change intervals, thereby reducing waste and expense. In this article, we realize a novel disposable sensor for moisture detection leveraging the material properties of the water absorbing polymer gel common to most diapers. We demonstrate a functional UHF RFID moisture monitor based on hydrogel sensing and propose a hybrid sensor design utilizing metal and hydrogel optimized for a specific diaper geometry. The proposed sensor design achieves a 1-meter read range, a bend radius of $<20 \mathrm{~mm}$, is insensitive to sensor orientation relative to the reader, and is lower-cost than sensors with metallic antennas. We detail design considerations for integration with manufacturing processes and conclude by exploring possible future applications enabled by hydrogel sensing.
\end{abstract}

\section{INTRODUCTION}

The advent of the Internet of Things and miniaturization of sensing devices has accelerated the growth of wearable health devices, with an estimated economic impact of $\$ 0.2 \mathrm{~T}$ by 2025 [1], [2]. Devices such as smart textiles and smartwatches are already used for real-time self-care and predictive healthcare applications by monitoring physiological conditions such as blood pressure, body temperature, respiration rate, and perspiration rate[3], [4]. As sensing, communication, and remote patient monitoring infrastructure become pervasive, the information contained in diaper waste may enable predictive health care and at-point diagnostics.

Urinary incontinence afflicts convalescent individuals and infants who are dependent on their caregivers. The elderly may suffer incontinence resulting from nerve deterioration or conditions including Alzheimer's or dementia. If diaper wearers are left unattended, they may suffer from rashes, discomfort, and bacterial infection. Diapers with embedded sensors could alert caregivers of when a diaper needs to be changed, increasing the quality of life of diaper wearers. The technology would ultimately save time and money by optimizing usage while reducing potential embarrassment for diaper wearers.
Diaper sensors have garnered research and industrial attention in a competitive marketplace [5], [6]. Developing multiparameter sensors is complex and costly, whereas a singleparameter sensor (moisture) could improve incontinence management and prevent health conditions at a lower cost.

Challenging the design of diaper moisture sensor is the low-cost target (diapers production costs under \$0.30/unit), complex diaper design, and high production rates. Ubiquitous, low-cost $(<\$ 0.10)$ RFID sensors lend themselves well to this application [7]. RFID-based sensors have been used for applications [8] in healthcare [9] for remote patient monitoring [10], as sensors for medical transfusion [11] and for haematocrit count [12]. RFID systems are bio-safe; however, associated sensing materials can increase system costs and may compromise on-body comfort.

In this paper, we propose a low-cost IoT-enabled smart diaper moisture system (Fig 1) designed with a passive RFID sensing mechanism. The RFID tag uses Super Absorbent Polymer (SAP), a subclass of hydrogel, as a body-safe, flexible sensing element. SAP is already the primary absorbent material in diapers. Using SAP as an antenna element can decrease the cost of RFID sensors by reducing the metal content and simplifying manufacturing and assembly. To the best of our knowledge, this is the first use of hydrogel as a functional element in antenna design. The contributions of this paper include:

- Designing, testing, and evaluating the design of a wearable, disposable, passive, metal-hydrogel based diaper moisture sensor

- Empirically demonstrating the use of hydrogel as an antenna element in RFID tags, sensitive to changes in moisture

- Discussion of diaper sensor design constraints and sensor manufacturability

The paper is organized as follows: Section 2 discusses related work on diaper moisture sensors, Section 3 identifies design requirements, and Section 4 describes the sensor development methodology including material characterization, sensor design, prototype fabrication, and validation. Section 5 lists the experimental results for evaluating sensor performance, and Section 6 explores future directions. 


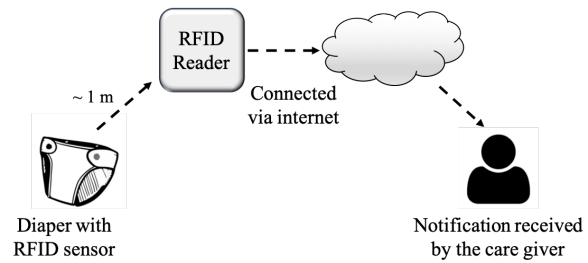

Fig. 1. Schematic visualizing the Internet of Things based smart diaper moisture detection system. Diaper is embedded with passive RFID Tag Antenna Based Sensor which communicates to RFID reader placed within the sensor read range, determined by the sensor geometry $(>1 \mathrm{~m})$. RFID reader is connected to the internet. Occurrence of an insult is detected by reader which triggers a notification to the care-giver or the diaper user as a message on smartphones or through other user interfaces

\section{RELATED WORK}

Many off-the-shelf diapers incorporate visual aids as wetness indicators[13], [14]. Visual indicators require proximity to the wearer and are not useful if the diaper is covered by a garment. Longer-range remote wireless sensors can help solve this problem. Remote wetness sensors can be categorized as external or internal relative to their placement on the diaper. Table I summarizes the operating principle of contemporary sensor designs. The sensor range is categorized as low $(<30$ $\mathrm{cm})$, medium ( $30 \mathrm{~cm}-5 \mathrm{~m})$, or high $(>5 \mathrm{~m})$.

External sensors are manually positioned to the outer diaper layer, after the diaper is put on the wearer. These sensors use external batteries to power long-range wireless technologies such as Bluetooth and Wi-Fi, increasing cost and bulk. Manual intervention required to remove and re-place sensor for every diaper change is an impediment to scalable deployment. Commercially available external sensors such as Diapersens [15] and others [16], [17], [18] use Bluetooth Low Energy (BLE) to pair with smartphones providing a theoretical read range of $30 \mathrm{~m}$. External sensor use requires a three-step manual process which includes hooking the sensor to diaper, covering the sensor with adhesive strips, and removing and cleaning the sensor after contamination. Sensor clean-up is unpleasant for many users. Additionally, sensor battery replacement and adhesive strips increase system cost. External sensors can ease the workload for individual adult caregivers at home but are not designed for large scale multi-bed hospital settings or daycares due to the labor-intensive changeover process.

Internal sensors are in-built to diapers and do not use external batteries making them less expensive and easier to deploy at scale. However, battery-less wireless technologies are limited by read-range. Tanaka et al. [19], [20], [21] have developed a sensor consisting of a urine-activated battery powering a wireless transmitter through an intermittent power supply circuit and a storage capacitor. The sensor transmits an ID when wet which when received generates a notification. The sensor provides a medium read range and can capture multiple events. However, response time is more than 5 minutes and the bulky design is challenging to incorporate with the existing diaper manufacturing lines. Using a multi-layer sensor design can also interfere with user-comfort and diaper absorption efficiency. [23], [24] have proposed sensor designs utilizing passive HF RFID which makes them inexpensive, but with a low read range $(<30 \mathrm{~cm})$ limiting their practical deployment.

There is a need to create a low-cost, wearable, disposable, and manufacturable internal sensor with a medium-high read range.

\section{UNDERSTANDING THE SENSOR ENVIRONMENT AND DESIGN REQUIREMENTS}

The first step towards designing a sensor requires an understanding of the sensing environment and design constraints. This section describes the diaper structure, its performance parameters, and diaper manufacturing processes. We subsequently outline target product requirements.

\section{A. Diaper structure and diaper performance}

Diapers consist of four functional layers, as shown in Fig 2. The top-sheet, a hydrophilic layer, enables rapid absorption of liquid to ensure skin stays dry. The distribution layer disperses the moisture to the layer's larger absorption volume. The third layer, the absorption layer, absorbs and retains the moisture inside the diaper. This is the thickest layer, consisting of Super Absorbent Polymer (SAP), a gel-like element contained within the fluff. Fluff, a non-woven material derived from cellulose, facilitates rapid absorption, whereas SAP provisions moisture retention under pressure, reducing leakage. The bottom-sheet is the fourth layer, a hydrophobic layer for repelling moisture and preventing seepage through the diaper [26]. Associated features such as fasteners, tapes, elastics, and leg cuffs ensure a good fit and prevent leakage.

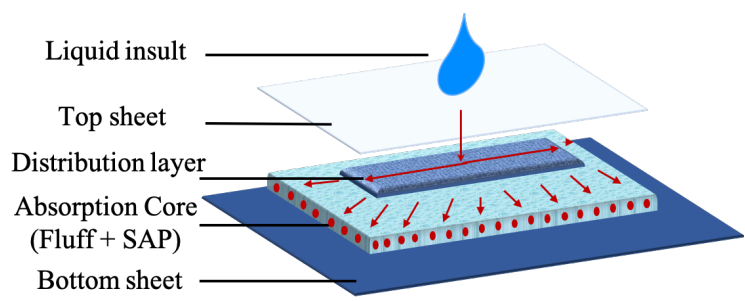

Fig. 2. The cross-section of a diaper and its constituent layers. Red arrows trace the spread and absorption of insult thorough the layers. The absorption layer, consisting of Super Absorbent Polymer and fluff, holds the bulk of the liquid absorbed by the diaper

Diaper performance is characterized by the rate of absorption, total capacity, water retention under pressure, and leakage resistance. International standards describe test methods to evaluate diapers and their performance[27]. Human factors also dictate that diapers are soft, comfortable, lightweight, aesthetically pleasing, and free of impurities to avoid skin rashes or other infections [28].

\section{B. Diaper manufacturing}

We met with representatives from a sponsoring diaper manufacturer to identify product needs. We toured their factory to understand how the machinery processes raw materials 
TABLE I

SUMMARY OF RELATED WORK ON DIAPER MOISTURE SENSORS

\begin{tabular}{|c|c|c|c|c|c|c|}
\hline $\begin{array}{l}\text { Sensor } \\
\text { Placement }\end{array}$ & Sensing Principle & $\begin{array}{l}\text { Wireless } \\
\text { Technology }\end{array}$ & Pros & Cons & Range & Reference \\
\hline \multirow{3}{*}{ External } & Printed visual stripes & NA & Cost; Manufacturability & Requires line-of sight & Low & [13] [14] \\
\hline & Proprietary technology & BLE & High sensitivity & $\begin{array}{l}\text { Sensor cost \& } \\
\text { maintenance }\end{array}$ & High & $\begin{array}{l}{[15][16]} \\
{[17]}\end{array}$ \\
\hline & $\begin{array}{l}\text { Temperature change at diaper } \\
\text { surface }\end{array}$ & BLE & High sensitivity & $\begin{array}{l}\text { Sensor size; } \\
\text { Cost }\end{array}$ & High & [18] \\
\hline \multirow{6}{*}{ Internal } & $\begin{array}{l}\text { Urine activated battery powering } \\
\text { RF Tx module }\end{array}$ & $\begin{array}{l}\text { Tx/Rx module } \\
\text { @ } 315 \mathrm{MHz}\end{array}$ & $\begin{array}{l}\text { Robustness; System captures } \\
\text { multiple instances }\end{array}$ & $\begin{array}{l}\text { Bulky design affecting } \\
\text { user-comfort; } \\
\text { Manufacturability; } \\
\text { Lag in response time }\end{array}$ & Medium & $\begin{array}{l}{[19][20]} \\
{[21]}\end{array}$ \\
\hline & $\begin{array}{l}\text { Modification of resonant frequency } \\
\text { between sensor tag and antenna }\end{array}$ & $\begin{array}{l}\text { RF inductive } \\
\text { coupling } \\
\text { @ } 12 \mathrm{MHz}\end{array}$ & Manufacturability & $\begin{array}{l}\text { Robustness; } \\
\text { Reader complexity }\end{array}$ & Low & [22] \\
\hline & $\begin{array}{l}\text { Sensing unit turns into voltage cell } \\
\text { activating a self-oscillator circuit for Tx }\end{array}$ & $\begin{array}{l}\text { Semi-passive } \\
\text { HF RFID }\end{array}$ & $\begin{array}{l}\text { Tag killed at disposal; } \\
\text { Transmits power only when } \\
\text { wet }\end{array}$ & $\begin{array}{l}\text { Complex sensor design } \\
\text { with two units; } \\
\text { Manufacturability }\end{array}$ & Medium & [23] \\
\hline & $\begin{array}{l}\text { Tag detected at reader due to increased } \\
\text { separation b/w a perforated metallic } \\
\text { sheet } \& \text { tag }\end{array}$ & $\begin{array}{l}\text { Passive } \\
\text { HF RFID }\end{array}$ & $\begin{array}{l}\text { Novel use of diaper material } \\
\text { (SAP) for displacement }\end{array}$ & $\begin{array}{l}\text { Metal sheet interferes } \\
\text { with user-comfort; } \\
\text { Robustness }\end{array}$ & Low & [24] \\
\hline & $\begin{array}{l}\text { Attenuation of signal strength received } \\
\text { at reader }\end{array}$ & $\begin{array}{l}\text { Passive } \\
\text { HF RFID }\end{array}$ & $\begin{array}{l}\text { Cost; } \\
\text { Simple design }\end{array}$ & Robustness & Low & [25] \\
\hline & $\begin{array}{l}\text { Increased signal strength at reader due } \\
\text { to increased antenna size when wet }\end{array}$ & $\begin{array}{l}\text { Passive } \\
\text { UHF RFID }\end{array}$ & $\begin{array}{l}\text { Simple design; Cost; } \\
\text { Robustness; lag-free }\end{array}$ & $\begin{array}{l}\text { Binary threshold } \\
\text { dry/wet detection }\end{array}$ & Medium & $\begin{array}{l}\text { Proposed } \\
\text { sensor }\end{array}$ \\
\hline
\end{tabular}

and finished diapers to ensure that our solution could be manufacturable at the cost, quantity, and rate required for economic viability and with minimal factory modification. The company asked that we not share specific information about their process. However, we can share that manufacturing occurs as part of a roll-to-roll process, materials are subject to high acceleration, tight bend radii, chemical adhesives, and high temperatures for a production rate greater than 500 diapers/minute. Lines are also regularly reconfigured to produce different diaper geometries, and waste material from imperfect production is shredded on-site and recycled for use in future diapers[29].

\section{Sensor design requirements}

We identified system requirements as listed in Table II based upon identified functional and operational constraints. A minimum read range of one meter between the sensor and the RFID reader is required to unlock use cases for practical deployment. Connecting RFID reader to cloud via secondary networks including $\mathrm{WiFi}$ can enable an extended range for end-device notification. Sensors must have a shelf-life of about three years to match that of disposable diapers. The sensor should be flexible for integration with manufacturing units and diaper packaging. The target for sensor material and assembly cost is to add under $25 \%$ to existing production costs to ensure the economic viability of the final product.

This work targets designing for the smallest diaper size (newborn, 0-3 months) to prove viability with the tightest constraints - a tag designed for the smallest diaper size can
TABLE II

SENSOR DESIGN REQUIREMENTS

\begin{tabular}{ll}
\hline Sensor requirement & Value \\
\hline System Range & $>1 \mathrm{~m}$ \\
Tag Dimensions & $<100 * 80 * 20 \mathrm{~mm}$ \\
Bend radius for packaging \& manufacturing & $<20 \mathrm{~mm}$ \\
Visible coloring & Neutral \\
Shelf-life & $>3$ years \\
Weight & $<10 \mathrm{~g}$ \\
Disposable & Yes \\
\hline
\end{tabular}

be scaled for larger adult diapers. The desired sensor output is binary (wet/dry) detection, and the sensor must be placed between absorption-layer and bottom-most layer to minimize interference with liquid absorption and retention. Tag safety and bio-safety are critical parameters and are discussed in the design section.

\section{SENSOR DESIGN AND DEVELOPMENT}

The RFID EPC Gen2 standard defines the protocol for communication between RFID readers and tags in the UHF band [30]. UHF RFID passive antenna systems are configured such that upon receiving the continuous wave sent by RFID reader, an IC alters the reflection co-efficient of tag antenna by varying its internal impedance, in-turn backscattering signal (an ID) to the reader. For maximum efficiency, the tag antenna is designed to be conjugately matched with input impedance of the tag chip. Off-the-shelf RFID tags available in the market are characterized to mostly operate in free-space environment. 
Tag Antenna Based Sensors (TABS) are passive RFID tags able to respond to changes in their surrounding environments [7]. An environmental state change affects the tag's electrical properties, which leads to an impedance variation, ultimately modifying properties of the sensor's returned signal. The RFID reader monitors the received signal and extracts the features relevant to characterize the sensor state. Notably, the impedance of a tag antenna placed inside a diaper will be affected by the surface impedance value of diaper material from both sides of the tag. The sensor design, therefore, must be sensitive to changes in dielectric behavior of wet and dry diaper material. We, thus, designed an experiment to characterize diaper materials' dielectric behavior. Material properties lay the foundations for creating sensor design hypotheses that satisfy the design requirements identified in section III. Design hypotheses include finding an antenna topology effectively correlating changes in the geometrical dimension of the antenna and its EM characteristics. Geometrical changes can consist of modifying the total length of the radiating element or the spacing between two dipole arms of the antenna. The creation of antenna design subsequently leads to the fabrication of the proposed sensor and experimental tests to validate the sensing hypothesis. These three sequential processes, as applied to our research, are described below.

\section{A. RF characterization of diaper material}

In section II, we identified that sensor will be placed between the diaper's bottom layer and third layer. TABS utilizes changes in the dielectric properties of the environment to determine the parameters reported by the diaper. Therefore, the first step in sensor design is to characterize the dielectric properties of diaper material for dry and wet states.

1) Dielectric properties of diaper material in dry and wet states: As shown in Fig 3, the test setup for characterizing material properties comprises of a planar resonant structure fabricated on a Taconic substrate, with its two ports connected to a Vector Network Analyzer (VNA). The resonant structure is formed of a transmission line coupled to a square-shaped complementary split ring resonator with a well-characterized frequency response resonant at $900 \mathrm{MHz}$. The diaper material sample used as material under test (MUT) is placed on splitring resonator (SRR). SRR is sensitive to permittivity or conductivity changes of the MUT due its small area and high confinement of electric fields. The working principle of using SRR includes measuring the relative shift of the notch position and depth of the transmission coefficient $\left(\mathrm{S}_{21}\right)$ on introduction of MUT [31].

The VNA is configured to capture the S-parameters of the MUT for a frequency sweep from $800 \mathrm{MHz}$ to $1 \mathrm{GHz}$, bandwidth utilized for UHF RFID tags in the microwave band [32]. In the context of RF engineering, scattering refers to the effect observed when plane electromagnetic wave passes across dissimilar dielectric media. For our setup which mimics a two-port network, S-parameters describe the electrical behavior of material in terms of incident, reflected and transmitted waves measured at the two ports. We are particularly

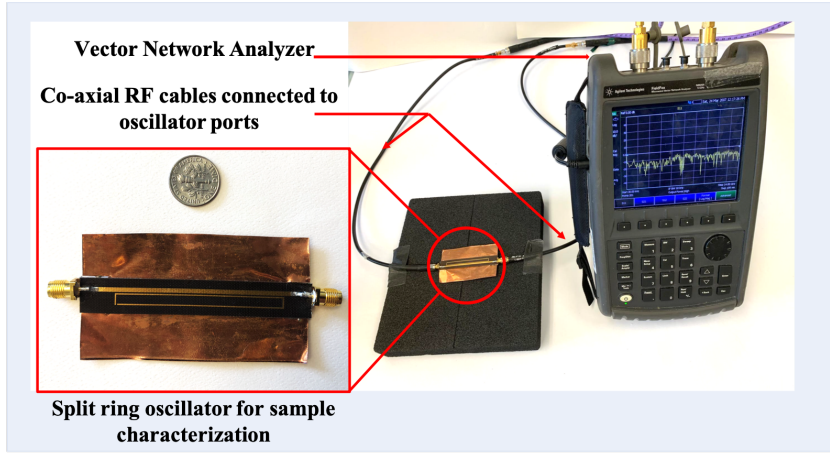

Fig. 3. The test setup used to characterize the dielectric behavior of diaper material mimicking a two-port network. Diaper material under test is placed on the Spilt Ring Oscillator and $0.9 \%$ saline solution is added in incremental quantities of $10 \mathrm{ml}$. A VNA captures the S-parameters of the MUT for the frequency sweep of $800 \mathrm{MHz}-1 \mathrm{GHz}$

interested in observing the behavior of the transmission coefficient between wet and dray state. A $0.9 \%$ saline solution is used as an industry standard as a substitute for baby urine for testing under laboratory conditions [33]. We captured the data for dry diaper material, and then for each incremental addition of $10 \mathrm{ml}$ of $0.9 \%$ saline solution until reaching $50 \mathrm{ml}$ total volume (saturation). Each state was allowed to absorb for two minutes to ensure a steady-state reading.

In order to maximize the absorption and minimize the chances of liquid leakage, diapers are thicker at the rear. For the diaper sample used in our experiments, we measured the thickness to vary from $4 \mathrm{~mm}$ in the front to $6.5 \mathrm{~mm}$ at the back. To eliminate differential behavior based on thickness, the experiment was repeated for two diaper samples with an average thickness of $4.5 \mathrm{~mm}$ and $5.5 \mathrm{~mm}$ front and rear, respectively.

figure 4 plots the transmission coefficient $S_{21}$ vs frequency (800 MHz -1 GHz) for two diaper samples of average thickness $4.5 \mathrm{~mm}$ (front of the diaper), and $5.5 \mathrm{~mm}$ (back of the diaper). The lack of distinguishable peak (or notch) at any frequency implies that diaper becomes a high-loss material when wet, which prevents us from calculating the precise dielectric constant. This could be attributed to presence of water layer in the diaper core. However, the change of amplitude and flattened bandwidth between $850 \mathrm{MHz}-930$ $\mathrm{MHz}$ (UHF band) indicates the conductivity of diaper core changes between dry/wet state in presence of saline liquids.

2) Estimation of relative change of conductivity of SAP when wet: Based of observations from subsection IV-A1 we suspected if the increase in conductivity could be attributed to presence of salt in water. However, literature review revealed that hydrogels change conductivity in presence of water [34]. Hydrogels are three-dimensional cross-linked hydrophilic polymer networks capable of swelling or de-swelling reversibly in water and retaining large volume of liquid in swollen state. Hydrogels' conductivity can be affected by method of synthesis, chemical structure, liquid concentration etc. Hydrogels and their biomedical applications such as drug 


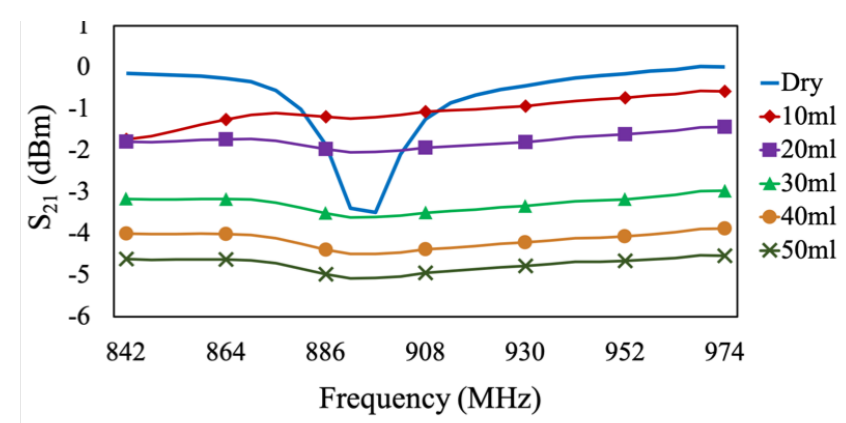

(i)

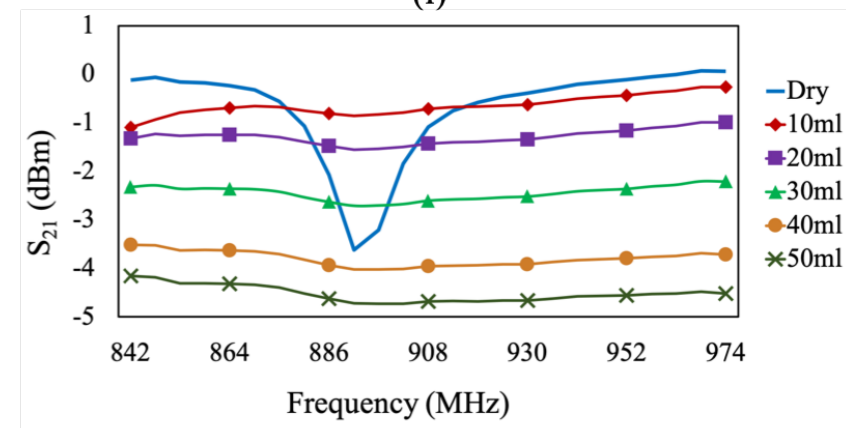

(ii)

Fig. 4. Plot of transmission co-efficient $\left(\mathrm{S}_{21}\right)$ vs frequency in dry state, and for every additional $10 \mathrm{ml}$ of $0.9 \%$ saline solution. As the diaper thickness varies from front to back, the plots are captured for average diaper thickness of (i) $4.5 \mathrm{~mm}$; (ii) $5.5 \mathrm{~mm}$ representative of front and back of diaper under test

delivery, tissue engineering, wound dressings, and hygiene products are an area of active research [35]. Since the diaper core consists of SAP (Section III-A), which is a sub-class of hydrogels [36], this electrical behavior extends to our sample too.

We designed an experiment to estimate the relative change in conductivity between dry and wet state for the particular polymer used in the MUT. The experiment was performed to verify if the conductive change is captured in the real environment repeatedly, and was not designed to be robust and sensitive to small variations. We 3D printed a $250 \mathrm{~mm}$ cube filled with $2 \mathrm{gm}$ of SAP, and inserted multimeter probes at the diagonal ends of the cube (figure 5). We captured the resistance under three states - dry SAP, with the addition of deionized water, and with addition of $0.9 \%$ saline solution. SAP acted as an insulator in dry state. When wet, SAP's conductivity was of the order of $10^{-2} \mathrm{~S} / \mathrm{m}$.

This section provides empirical evidence that diaper core, the sensing environment, becomes conductive when wet. Further, SAP-which is present in the absorption core of diaperhas conductivity of the order of $10^{-2} \mathrm{~S} / \mathrm{m}$, which should measurably impact signal properties.

\section{B. Hypothesis for sensing mechanism and sensor design}

The next step in sensor development includes hypothesizing the sensing principle, selecting the antenna form, and selecting the reader's sensing parameter.

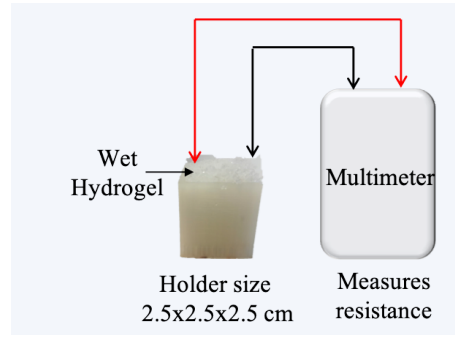

Fig. 5. This setup estimated the relative change of SAP conductivity in dry/wet states. Multimeter probes are inserted diagonally in a 3-D printed cube of dimension $2.5 \times 2.5 \times 2.5 \mathrm{~cm}$ filled with $2 \mathrm{gm}$ of dry SAP. Resistance was measured in (i) the dry state (ii) when wetted with deionized water (iii) when wetted with $0.9 \%$ saline solution

We hypothesized that a change in conductivity of SAP makes it a suitable material for antenna design. As the tag wets, the hydrogel will become conductive, and its inclusion in the tag antenna will increases the power supplied to the RFID chip, since conductive surface area of antenna increases. As passive tags are forward-link limited, providing sufficient power to the IC nearly assures a back-scatter response. Further, using in-situ diaper material as an antenna sense element eliminates the risk posed by environmental variability.We looked for examples demonstrating the use of hydrogels as antenna elements in RFID tags. In [37], Amendola et al. discuss the possibility of using biocompatible hydrogels as a sensing substrate for RFID based smart plasters because of change in RF dielectric properties on the absorption of epidermal fluids. However, the use of hydrogels as a component of tag antenna design has not previously been demonstrated.

The decision on antenna topology was guided by the need to have a simple antenna configuration capable of increasing the size of the radiating element when wet. Additionally, the sensor should be insensitive to motion and orientation changes. These factors led us to choose bow-tie antennas, as they are known for robust and stable performance over a wide frequency range despite their simple design [38][39]. The dimensions of the bow-tie antenna were kept within the boundary conditions identified in Section III.

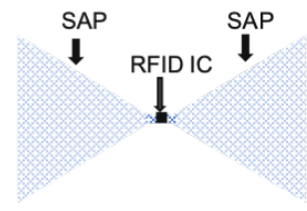

(i)

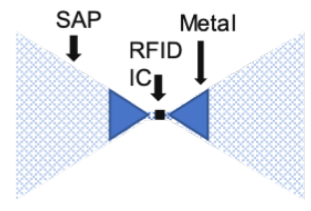

(ii)
Fig. 6. Sensor design illustration used as reference for sensor fabrication; (i) Only hydrogel based tags (ii) Hydrogel-metal hybrid tags

The conductivity of SAP is markedly less than Copper or other metals which are used for making tag antennas. For the lack of baseline data on the performance of hydrogel based tags, we used an experimental approach of testing tag designs with varying content of metal and hydrogel. Metallic presence 
reduces the uncertainty associated with tag performance and improves the likelihood of the reader receiving a response. We conceptualized the design of hybrid tags, as shown in figure 6 ,to be used as a reference for fabrication.

\section{Development of prototype and hypothesis validation}

Using the sensor design from above, we hand-fabricated six sensors on a plastic substrate (Mylar PET). Sensor design had incremental metal content from no copper to $30 \%$ of tag area. Each sensor was placed centrally between the base sheet and the absorption core. Diapers with sensor were put on RFpermeable dolls filled with saltwater to emulate the human body's presence. figure 7 shows the schematic of the test setup used for sensor performance and hypothesis validation. A RFID reader placed at a distance of $30 \mathrm{~cm}$ from the diaper wearing doll continuously logged received power (RSSI) to capture the change between dry and wet state upon the addition of $60 \mathrm{ml}$ of $0.9 \%$ saline solution. The saline solution was poured onto the diapers using a syringe connected to a tube opening to diaper center. Typically, a newborn baby weighing about $3 \mathrm{~kg}$ releases about $30 \mathrm{ml}$ of discharge per insult event. We selected $60 \mathrm{ml}$ as the discharge amount to represent the upper limit at which the diaper should be changed, aligning with the standard absorption tests performed in industry. Table III captures the dry/wet power received by the reader at a distance of $30 \mathrm{~cm}$. The sensor range is the distance at which the reader stops detecting the signal sent by the doll while moving away from it. Figure 8 shows the fabricated hybrid sensor design in the dry and wet state.

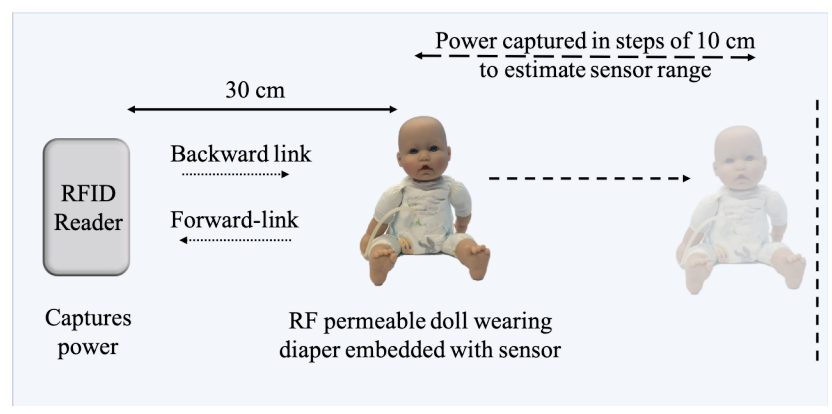

Fig. 7. A schematic of the test setup used for recording the change in RSSI at the reader in dry and wet ( $60 \mathrm{ml}$ of $0.9 \%$ saline solution) state. A diaperwearing RF permeable doll was initially kept at $30 \mathrm{~cm}$ from the reader. Sensor range was determined by capturing power at every increase of $10 \mathrm{~cm}$ in the wet state until the tag became unresponsive

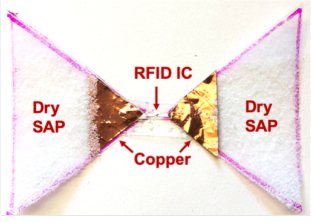

(i)

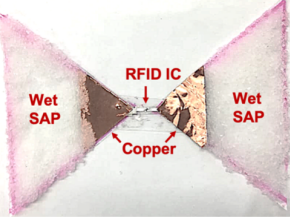

(ii)
Fig. 8. Fabricated hybrid tags with part copper and part hydrogel on the bow-ties in (i) dry state; (ii) wet state
TABLE III

RSSI RECEIVED AT READER IN DRY AND WET STATE OF SENSOR WITH DIMENSION $<100 * 80 * 20 \mathrm{MM}$

\begin{tabular}{ccccc}
\hline $\begin{array}{c}\text { Tag } \\
\text { number }\end{array}$ & $\begin{array}{c}\text { \% by area of } \\
\text { Copper }\end{array}$ & $\begin{array}{c}\text { RSSI when dry } \\
(\mathrm{dBm})\end{array}$ & $\begin{array}{c}\text { RSSI when wet } \\
(\mathrm{dBm})\end{array}$ & $\begin{array}{c}\text { Range } \\
(\mathrm{cm})\end{array}$ \\
\hline Tag 1 & 0 & NA & -50 & 30 \\
Tag 2 & 1.48 & -58 & -48 & 50 \\
Tag 3 & 4.74 & -54 & -42 & 80 \\
Tag 4 & 12.44 & -53 & -41 & 100 \\
Tag 5 & $\mathbf{2 0 . 1 5}$ & $\mathbf{- 5 1}$ & $\mathbf{- 3 8}$ & $>\mathbf{1 0 0}$ \\
Tag 6 & 28.89 & -50 & -37 & $>150$ \\
\hline
\end{tabular}

The data captured in Table III illustrates the trade-offs between cost, range, and performance. All tags excluding Tag 1 show a difference of $10-12 \mathrm{dBm}$ between dry and wet states. The power received in dry and wet states increments while moving from Tag 1 to Tag 6 as the amount of Copper increases. A similar trend is observed in the measured range. Tag 5 achieves the desired range required for the system. Addition of Copper while moving from Tag 5 to Tag 6 will also add to the extra tag cost. We chose Tag 5 as the final sensor design for further testing. Notably, Tag 1 , which is made only of the hydrogel, demonstrates a range of $30 \mathrm{~cm}$ with a power level of $-50 \mathrm{dBm}$ in the wet state. This, we believe, is the first demonstration of a functional tag designed using only hydrogels.

Note that there are other tradeoffs to consider in designing diaper sensor which may be difficult to quantify. For example, body-safety is paramount. Copper is considered safe and a necessary element for wellness and for human-skin [40]. Copper can also be replaced with silver, which is known to have anti-microbial properties [41]. Being metallic, the electrical behavior of silver is similar to copper, so no redesign of sensor design will be necessary - though cost will increase.

Tag disposal and safety are other important lifecycle criteria. Used diapers should stop being responsive to electronic interrogation signals to avoid interference with active tags and other disposal activities. RFID EPC Gen2 standard specifies a kill command that may be used to deactivate discarded tags (dirty diapers) [30]. This command could be issued automatically by an end-use application once the diaper reaches specified wetness. Regarding radiation safety, the U.S. FDA has declared RFID as safe in healthcare settings [42], with radiation limits stating that the reader would need to be within $10 \mathrm{~cm}$ of a human to reach a harmful level [43].

\section{EXPERIMENTAL RESULTS AND DISCUSSION}

In section IV-C, we identified a hybrid metal-SAP sensor design which satisfies the binary detection of moisture in the diaper. This section evaluates the performance for the identified sensor size in real-operating conditions, with results presented below.

\section{A. Sensor Reliability}

We prototyped and tested 10 nominally-identical sensors. Using the experimental setup shown in figure 7, we captured 
the RSSI value in the dry state and upon the addition of $60 \mathrm{ml} 0.9 \%$ saline solution. Figure 9 shows the plot of the RSSI versus time as the sensor moves from the dry to wet state. The average value over 10 samples increases by 13 $\mathrm{dBm}$ from $-53 \mathrm{dBm}$ to $-40 \mathrm{dBm}$. The figure also shows the values one standard deviation and below the average. The variation in RSSI may be attributed to hand-fabrication of sensor designs or component-level variations, which outlines the need for tight process controls for sensor fabrication. The broader variation in the wet state may be attributed to the uneven distribution of liquid inside the diaper, which is nonrepeatable due to manufacturing variability.

We subsequently conducted an unequal variances t-test to compare the RSSI in the dry state and wet state. There is a difference in the signal strength for dry state $(M=-53.79$, $\mathrm{SD}=3.26)$ and wet state $(\mathrm{M}=-40.67, \mathrm{SD}=-4.62) ; \mathrm{t}(16)=7.32$, $\mathrm{p}$ $=0.005$. These results statistically confirm that signal strength is greater in the wet state than in the dry state.

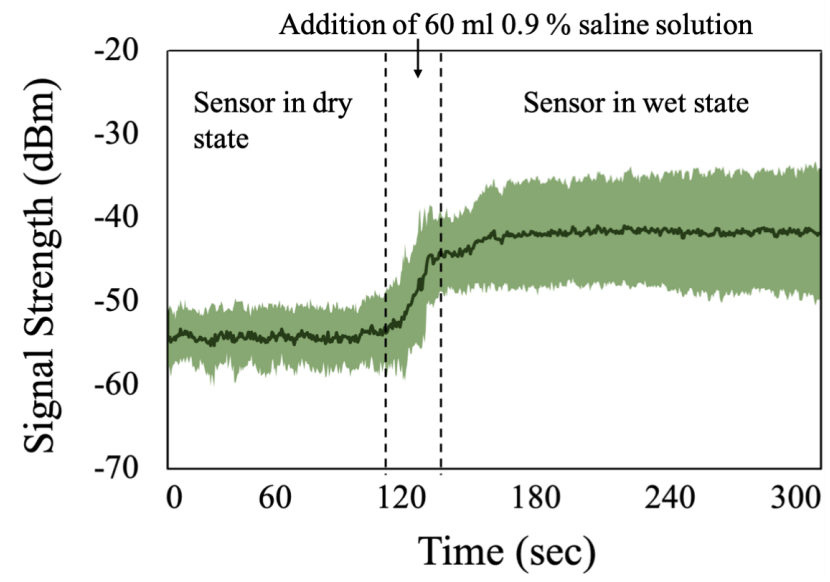

Fig. 9. The plot of RSSI captured at the reader as the sensor transitions from dry to wet state on the addition of $60 \mathrm{ml}$ of $0.9 \%$ saline solution. The dark line shows the average value over ten samples, and the boundary of the lighter portion shows the upper bound and lower bound values using one standard deviation. The average strength increases by $13 \mathrm{dBm}$ when state transitions from dry to wet

Notably, the sensor provides an instantaneous response to the addition of insult, enabling real-time monitoring. The system takes only 30 seconds to reach a stable reading state in the wet state. Further, placing the sensor in the middle, right below the insult location, ensures that liquid insult will always contact the sensor and make it wet.

\section{B. Range}

Achieving a read range of at least 1 meter from a RFID reader connected to the cloud is a critical performance metric. Figure 10 shows the plot of RSSI value captured for $10 \mathrm{~cm}$ increases in distance between the tag and reader; using the setup shown in figure 7. A read range of $100+\mathrm{cm}$ was achieved reliably for all tested samples. An increase of metallic content in a hybrid sensor of the same dimensions improves the read range beyond 1 meter.

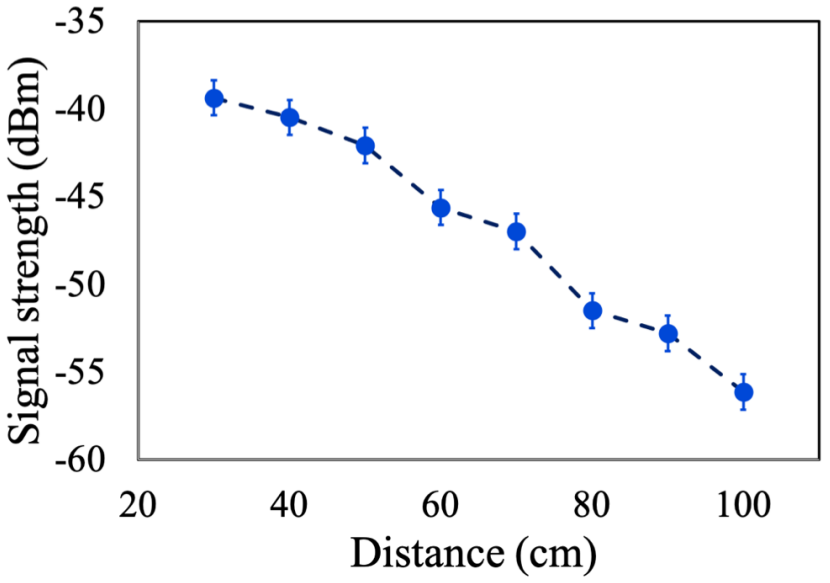

Fig. 10. The plot of average RSSI captured at the reader vs. the distance between the diaper sensor and RFID reader. The measurement is taken for diapers with sensors put on RF permeable dolls, soaked in $60 \mathrm{ml} 0.9 \%$ saline solution

\section{Flexibility and reader orientation}

The power received by the tag and the backscatter power received at the reader may drastically vary as a function of relative orientation between tag and reader. Diaper wearers are not static; hence, the system needs to be insensitive to wearer orientation and movements. We tested for power received at the reader for a change in doll's position from lying on the back to sitting upright, shown in figure 11 (i) \& (ii). The power received while lying down decreases only by $2 \mathrm{dBm}$, likely as the baby's weight spreads over a larger area changing diaper's compression (figure 12 (i)), establishing insensitivity to reader-tag orientation.

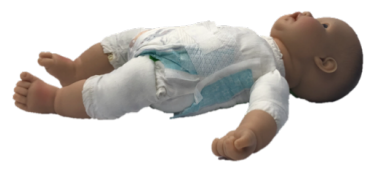

(i)

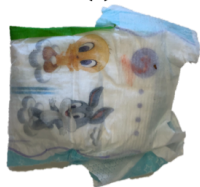

(iii)

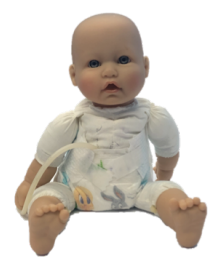

(ii)

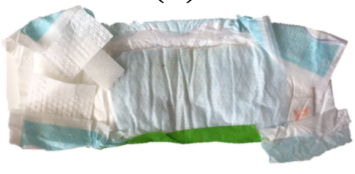

(iv)
Fig. 11. The doll wearing wet diaper (i) lying on the back (ii) sitting; Dry diaper with sensor when (iii) folded and (iv) unfolded. Testing the doll with the diaper in different positions establishes independence with respect to the reader's orientation, making the design robust for practical usage. Testing a dry diaper with sensor folded/unfolded validates the diapers' pack-ability

Tag flexibility is also an essential characteristic as diapers are folded from the center for packaging and selling to the end customers. A functional verification of sensor flexibility was performed by capturing the power when the dry diaper 
is folded and unfolded, as shown in figure 11 (iii) \& (iv). A negligible change of $1 \mathrm{dBm}$ is observed (Figure 12 (ii)), fulfilling the practical constraint of pack-ability.

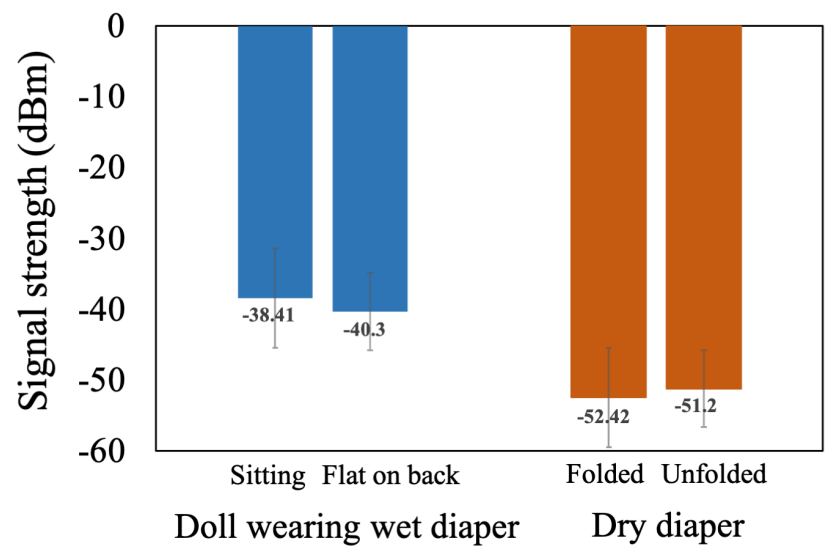

Fig. 12. Bar graphs for power received at reader kept $30 \mathrm{~cm}$ away from the measurand (i) RF permeable doll wearing diaper lying on its back and in sitting position for the wet state (ii) Diaper folded and unfolded in dry state The minimal changes in received power establish design robustness

These experiments validate the independence between reader-tag orientation and tag flexibility, validating the robustness of the system. That the tag fulfills the condition for sensor bend radius to be $<20 \mathrm{~mm}$ is an important first step towards including it in existing manufacturing systems. Additionally, the sensor placed in the diaper is invisible to the naked eyes, which preserves the original look and feel of the diaper, easing acceptance.

\section{Cost and Manufacturability}

Traditionally, metal based RFID tags have been manufactured using copper etching techniques. Research has shown that paper based inkjet printed RFID tags give good performance while providing enhanced flexibility to the antenna design [44], [45]. For a hybrid sensor, SAP could be sprayed onto the diaper substrate and metal components could be added by inkjet printing. Such changes can be added to the existing manufacturing units with minimal modifications, and therefore the the delta in cost is negligible when amortized across the volume of diapers produced over the equipment's useful life. The tag cost will also be minimized by using preexisting diaper material, as opposed to adding metal.

\section{E. Design reusability}

Varied real-life applications require different performance parameters from the sensor. A smaller range is suited for static deployment in a multi-bed hospital. In contrast, an extended range is needed for use by the elderly moving in care homes. The desired sensor performance can be achieved by altering two design parameters (i) overall sensor dimension (ii)\% metal content present on the bow-tie antennas. Adult diapers are larger and capable of incorporating larger sensors. Increasing the sensor dimension or \% metal content on bow-tie will increase the sensor range and vice versa. Thus, the hybrid approach provides flexibility and reusability to sensor design so that performance can be determined by the envisioned application and associated cost-range trade-offs.

The tests performed in this section provide evidence that the designed sensor meets the requirements identified in II. In this paper, the sensor is designed for binary wet/dry detection only, with sensor behavior characterization restricted to 60 $\mathrm{ml}$ of release in a baby diaper. Further characterization may allow us to change the trigger for the wet state to a desired threshold level, and to determine wetness levels with improved precision.

\section{CONCLUSIONS AND FUTURE WORK}

The market for wearable health devices is gaining traction. Interest in smart diapers is compounded by providing improved safety, security and freedom of movement to users, and also the potential for enabling point-of-care healthcare diagnostics. To-date, an inexpensive, robust, diaper-integral moisture sensor has been unavailable because of complexity of diaper design and low manufacturing cost targets. We propose and empirically demonstrate a wearable passive RFID moisture sensor which re-purposes contemporary diaper materials, namely Super Absorbent Polymer, as its sensing element. This is a novel use of hydrogel as a functional antenna element in the UHF range.

In the paper, we performed a study of diaper structure and its manufacturing facilities and identified sensor design requirements. We then illustrated the sensor development stages and results. The first stage included characterizing the dielectric properties of diaper material which helped us to conclude that SAP, material responsible for the majority of absorption in diapers, increases its conductivity to the order of $10^{-2} \mathrm{~S} / \mathrm{m}$ when wet. We rationalized that the material conductivity of hydrogel when wet could be leveraged for sensing and as a functional antenna element for tag design. We hypothesized that a bow-tie antenna design composed of metal and SAP would increase in size when exposed to an insult, leading to increased power supplied to RFID tag chip, and therefore heightened backscatter power when wet. We validated this hypothesis by prototyping the proposed sensor design and evaluated the trade-off in sensor performance by relating metal content to the range, selecting a tag with $20 \%$ metal content as an appropriate balance of range (exceeding $1 \mathrm{~m}$ ) and cost. This sensor proved repeatable, reliable, flexible, body-safe, and disposable, and should be manufacturable using inkjetting technologies and scalable to larger diaper geometries.

Future work will aim to provide more granular moisture detection, with an ability to detect amount of insult passed each time. This can be accomplished by placing another sensor in the tag to serve as a ground-truth reference, which also helps to disambiguate moisture readings from distance by comparing RSSI measurements. Data collected from tracking the diaper moisture can provide early warnings against dehydration, urinary tract infection or even loss of kidney functions. The sensors can be integrated with a mobile phone application or as 
a part of the smart home applications for predictive ordering, or voice assistants to ease status checking.

The use of hydrogels in biomedical applications is an area of active research with applications noted in drug delivery, wound dressings, hygiene products, among others. Demonstrating hydrogels' use as an element capable of sensing and transmission could enable further exploration of its diagnostic capabilities, for example, characterizing the change in $\mathrm{pH}$ of SAP in the presence of abnormal urine, or glucose sensing for monitoring diabetes. The research published in this paper could lead to new multi-modal sensing capabilities on passive RFID tags using hydrogels, an important step to enable the Medical Internet of Things and to build a smarter connected world where sensors are so low cost that theyre disposable, so pervasive that we have ubiquitous data, and such information richness that we can one day sense impending health conditions.

\section{ACKNOWLEDGEMENT}

This research was funded by Softys under the MIT Industry Liaison Program. We are thankful to the sponsors for explaining the intricacies of the diaper structure, diaper performance testing, and for showing us the manufacturing lines. These discussions were crucial for us to identify the constraints for sensor design and sense environment.

\section{REFERENCES}

[1] S. C. Mukhopadhyay, "Wearable sensors for human activity monitoring: A review," IEEE sensors journal, vol. 15, no. 3, pp. 1321-1330, 2014.

[2] J. Manyika, The Internet of Things: Mapping the value beyond the hype. McKinsey Global Institute, 2015.

[3] D. Dias and J. Paulo Silva Cunha, "Wearable health devicesvital sign monitoring, systems and technologies," Sensors, vol. 18, no. 8, p. 2414, 2018.

[4] M. Haghi, K. Thurow, and R. Stoll, "Wearable devices in medical internet of things: scientific research and commercially available devices," Healthcare informatics research, vol. 23, no. 1, pp. 4-15, 2017.

[5] M. McKnight, F. Lin, H. Kausche, T. Ghosh, and A. Bozkurt, "Towards paper based diaper sensors," in 2015 IEEE Biomedical Circuits and Systems Conference (BioCAS). IEEE, 2015, pp. 1-4.

[6] B. Pepin and H. Yao, "Smart diaper for detecting and differentiating feces and urine," April 2018, uS Patent App. 15/292,389.

[7] R. Bhattacharyya, C. Floerkemeier, and S. Sarma, "Low-cost, ubiquitous rfid-tag-antenna-based sensing," Proceedings of the IEEE, vol. 98, no. 9, pp. 1593-1600, 2010.

[8] X. Jia, Q. Feng, T. Fan, and Q. Lei, "Rfid technology and its applications in internet of things (iot)," in 2012 2nd international conference on consumer electronics, communications and networks (CECNet). IEEE, 2012, pp. 1282-1285.

[9] L. Antti, "A short overview of the rfid technology in healthcare," in Systems and Networks Communications. ICSNC'09. Fourth International Conference on, 2009.

[10] A. Cangialosi, J. E. Monaly, and S. C. Yang, "Leveraging rfid in hospitals: Patient life cycle and mobility perspectives," IEEE Communications Magazine, vol. 45, no. 9, pp. 18-23, 2007.

[11] Z. Jiang, Z. Fu, and F. Yang, "Rfid tag antenna based wireless sensing method for medical transfusion applications," in 2012 IEEE International Conference on RFID-Technologies and Applications (RFID-TA). IEEE, 2012, pp. 126-130.

[12] R. Bhattacharyya, E. Amin, I. Ehrenberg, S. Sarma, C. Swanson, B. Tien, and M. Wong, "Towards low-cost, wireless blood anomaly sensing: An rfid-based anemia detection sensor," in 2015 IEEE International Conference on RFID (RFID). IEEE, 2015, pp. 189-196.

[13] Procter and Gamble. Pampers swaddlers. [Online]. Available: https://www.pampers.com/en-us/diapers-wipes/pampersswaddlers-diapers
[14] Kimberly-Clark. Huggies little snugglers. [Online]. Available: https://www.huggies.com/en-us/diapers/little-snugglers

[15] DiaperSen. Diapersens adult incontinence smart diaper sensor for wet diaper alert. [Online]. Available: "https://www.amazon.com/DiaperSensAdult-Incontinence-Diaper-Sensor/dp/B07DBH8KXK"

[16] O'PRO9. O'pro9 smart wearable humidity sensor instant alert notification reminder prevent adult baby diaper rash. [Online]. Available: https://amzn.to/2XtP5Ae

[17] Geecare. Geecare Thin Smart Baby Wet Diaper Alarm Monitoring Baby Urine Regularity and Lost Prevent with Mobile Phone APP. [Online]. Available: https://amzn.to/2LFEaS2

[18] T. Khan, "A smart wearable gadget for noninvasive detection and notification of diaper moister," in 2018 IEEE International Conference on Electro/Information Technology (EIT). IEEE, 2018, pp. 0240-0244.

[19] A. Tanaka, T. Yamanaka, H. Yoshioka, K. Kobayashi, and T. Douseki, "Self-powered wireless urinary incontinence sensor for disposable diapers," in SENSORS, 2011 IEEE. IEEE, 2011, pp. 1491-1494.

[20] A. Tanaka, F. Utsunomiya, and T. Douseki, "Wearable self-powered diaper-shaped urinary-incontinence sensor suppressing response-time variation with 0.3-v start-up converter," in SENSORS, 2014 IEEE. IEEE, 2014, pp. 684-687.

[21] A. Tanaka, R. Suematsu, H. Sakamoto, and T. Douseki, "Self-powered wireless urinary-incontinence sensor determines time for diaper change from spacing between sensing signals," in 2016 IEEE SENSORS. IEEE, 2016, pp. 1-3.

[22] L. Yambem, M. K. Yapici, and J. Zou, "A new wireless sensor system for smart diapers," IEEE Sensors Journal, vol. 8, no. 3, pp. 238-239, 2008.

[23] J. Sidén, A. Koptioug, and M. Gulliksson, "The" smart" diaper moisture detection system," in 2004 IEEE MTT-S International Microwave Symposium Digest (IEEE Cat. No. 04CH37535), vol. 2. IEEE, 2004, pp. 659-662.

[24] M. A. Ziai and J. C. Batchelor, "Smart radio-frequency identification tag for diaper moisture detection," Healthcare technology letters, vol. 2, no. 1, pp. 18-21, 2015.

[25] K. Yamada, N. Toshiaki, K. Ishihara, Y. Ohno, A. Ishii, S. Shimizu, T. Araki, R. Takahashi, H. Takahashi, and E. Shimizu, "Development of new type incontinence sensor using rfid tag," in 2010 IEEE International Conference on Systems, Man and Cybernetics. IEEE, 2010, pp. 26952700.

[26] S. Dey, M. Purdon, T. Kirsch, H. Helbich, K. Kerr, L. Li, and S. Zhou, "Exposure factor considerations for safety evaluation of modern disposable diapers," Regulatory Toxicology and Pharmacology, vol. 81, pp. 183-193, 2016.

[27] I. S. Organization(ISO). Iso 15621:2017(en): Absorbent incontinence aids for urine and/or faeces general guidelines on evaluation. [Online] Available: https://www.iso.org/obp/ui/\#iso:std:iso:15621:ed-3:v1:en

[28] C. White, "Engineered structures for use in disposable incontinence products," Proceedings of the Institution of Mechanical Engineers, Part H: Journal of Engineering in Medicine, vol. 217, no. 4, pp. 243-251, 2003.

[29] T. Machinery. What is baby diapers manufacturing process. [Online]. Available: http://www.tmxmachinery.com/diapermaking-machine/baby-diaper-making-machine/what-is-baby-diapersmanufacturing-process.html

[30] G. EPCglobal, "Epc radio-frequency identity protocols generation-2 uhf rfid; specification for rfid air interface protocol for communications at 860 mhz-960 mhz," EPCglobal Inc., November, 2013.

[31] K. Saeed, R. D. Pollard, and I. C. Hunter, "Substrate integrated waveguide cavity resonators for complex permittivity characterization of materials," IEEE Transactions on Microwave Theory and Techniques, vol. 56, no. 10, p. 2340, 2008.

[32] G. EPCglobal. (2019, March) Regulatory status for using rfid in the epc gen2 (860 to $960 \mathrm{mhz}$ ) band of the uhf spectrum. [Online]. Available: https://www.gs1.org/docs/epc/uhf_regulations.pdf

[33] E. the voice of nonwovens, "Edana guidelines for the testing of baby diapers," April 2016.

[34] P. Pissis and A. Kyritsis, "Electrical conductivity studies in hydrogels," Solid State Ionics, vol. 97, no. 1-4, pp. 105-113, 1997.

[35] E. Caló and V. V. Khutoryanskiy, "Biomedical applications of hydrogels: A review of patents and commercial products," European Polymer Journal, vol. 65, pp. 252-267, 2015. 
[36] E. M. Ahmed, "Hydrogel: Preparation, characterization, and applications: A review," Journal of advanced research, vol. 6, no. 2, pp. 105$121,2015$.

[37] S. Amendola, C. Occhiuzzi, A. Ajovalasit, M. Sabatino, C. Dispenza, and G. Marrocco, "Dielectric characterization of biocompatible hydrogels for application to epidermal rfid devices," in 2015 European Microwave Conference (EuMC). IEEE, 2015, pp. 379-382.

[38] C. A. Balanis, Antenna theory: analysis and design. John wiley \& sons, 2016.

[39] M. Nikkari, T. Bjorninen, L. Ukkonen, F. Yang, A. Elsherbeni, L. Sydanheimo, and M. Kivikoski, "Design and comparison between two general purpose dipole type uhf rfid tag antennas," in 2008 IEEE Antennas and Propagation Society International Symposium. IEEE, 2008, pp. 1-4.

[40] G. Borkow, "Using copper to improve the well-being of the skin," Current chemical biology, vol. 8, no. 2, pp. 89-102, 2014.

[41] A. B. Lansdown, "A pharmacological and toxicological profile of silver as an antimicrobial agent in medical devices," Advances in pharmacological sciences, vol. 2010, 2010.

[42] U. Food and D. Administration. Radio frequency identification (rfid). [Online]. Available: "https://www.fda.gov/radiationemitting-products/electromagnetic-compatibility-emc/radio-frequencyidentification-rfid"

[43] bharath. (2018, May) Does uhf rfid pose a radiation threat to people? [Online]. Available: https://www.rfidjournal.com/blogs/experts/entry?12027

[44] Y. Amin, S. Prokkola, B. Shao, J. Hallstedt, H. Tenhunen, and L.R. Zheng, "Inkjet printed paper based quadrate bowtie antennas for uhf rfid tags," in 2009 11th International Conference on Advanced Communication Technology, vol. 1. IEEE, 2009, pp. 109-112.

[45] A. Rida, L. Yang, and M. M. Tentzeris, "Design and characterization of novel paper-based inkjet-printed uhf antennas for rfid and sensing applications," in 2007 IEEE Antennas and Propagation Society International Symposium. IEEE, 2007, pp. 2749-2752. 